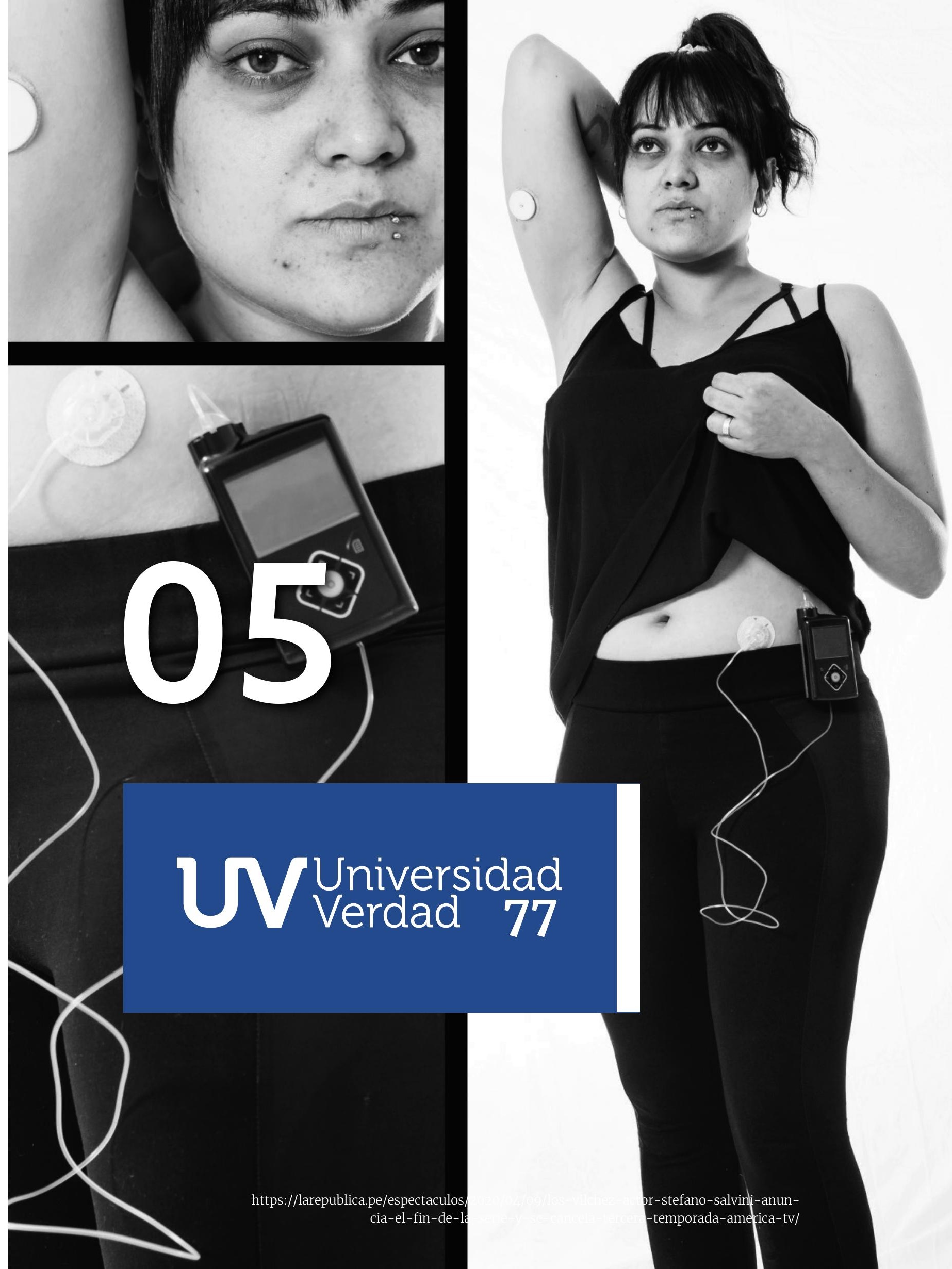




\title{
CUERPOS QUE HABLAN: CONSTRUCCIÓN DE NARRATIVAS DEL CUERPO ENFERMO, A PARTIR DE LAS ESTÉTICAS DE LA DISIDENCIA
}

\section{Bodies that speak construction of narratives of the sick body, based on the aesthetics of dissent}

\author{
iD Paulo Freire Valdiviezo, Universidad de Cuenca. (Ecuador) \\ (paulo.freire@ucuenca.edu.ec) (https://orcid.org/0000-0001-5370-0033) \\ iD Verónica Neira Ruiz, Universidad del Azuay. (Ecuador) \\ (veronicaneirar@uazuay.edu.ec) (https://orcid.org/0000-0002-3407-6893)
}

\section{Resumen}

En este artículo se abordan los resultados de la investigación y sus reflexiones en torno a la posibilidad de construir narrativas del cuerpo enfermo a partir del diálogo con personas que viven con enfermedades crónicas visibles. El objetivo es discutir sobre la construcción de nuevas narrativas en salud que rompan con la narrativa normalizada de la relación entre estética y cuerpo sano. El abordaje metodológico de esta investigación tiene un enfoque cualitativo y ha sido pensado desde el análisis etnográfico para la descripción de una realidad concreta. Las técnicas de investigación aplicadas fueron entrevistas a profundidad, grupos focales y la fotografía como técnica de construcción narrativa. Además del análisis sobre los relatos de los participantes en la investigación, se trabajó en la construcción de una secuencia fotográfica que presente en la construcción de una narrativa del cuerpo enfermo y de la exploración de las posibilidades estéticas de las corporalidades disidentes. Finalmente, este trabajo plantea la relación que existe entre los imaginarios normalizados del cuerpo sano y estéticamente bello, con las categorías de etnia y clase presente en los discursos sobre la estética dominante en la ciudad de Cuenca.

\section{Abstract}

This article addresses the reflections on the possibility of constructing narratives of the sick body, based on a dialogue with people who live with chronic diseases. The objective of the article is to discuss the construction of new narratives that break with the normalized narrative of the relationship between aesthetics and the healthy body. The methodological approach of this research is qualitative and has been drawn from ethnographic analyses for the description of a concrete reality. The research techniques that were applied are indepth interviews, focus groups, and photography as a narrative construction technique. In addition to the analysis of the stories of the participants in the research, this article presents the construction of a photographic sequence that works as the construction of a narrative of the sick body and the exploration of the aesthetic possibilities of dissident corporeality. Finally, this paper intervenes on the relationship that exists between the normalized imaginaries of healthy and aesthetically beautiful bodies, with the categories of ethnicity and class present in advertising discourses.

\section{Palabras clave}

Cuerpo, disidencia, enfermedad, narrativa, normalización, salud.

Keywords

Body, dissent corporeality, chronic disease, narrative, normalization, health.. 
1.

\section{Introducción}

El cuerpo es un tema de discusión recurrente en las ciencias sociales; su control se ha convertido en motivo de elaboración de políticas públicas. Los planes de salud y los currículos educativos son construidos teniendo en cuenta el cuerpo como un eje central. Vigilar y castigar, como diría Foucault (1983), fue un imperativo para moldear los cuerpos de acuerdo a constructos políticos, ideológicos, morales y religiosos dominantes.

Pero al hablar de cuerpo, tenemos que ir más allá del objeto que ocupa un espacio material; al hablar de cuerpo tenemos que hacer mención del discurso que este encierra y proyecta; por este motivo, analizaremos el cuerpo como un dispositivo discursivo, es decir, que miran al cuerpo en términos de multiplicidad ya que en él convergen varios elementos de ideología, política, economía (Deleuze, 1989), capaz de acoger la mirada política de una sociedad.

La construcción discursiva de los cuerpos encierra parámetros estéticos alineados con una visión homogénea de la masculinidad, de la feminidad y de la ciudadanía. En otras palabras, estaríamos hablando de lo que Butler (2012) ha denominado "cuerpos que importan", pues, socialmente se ha construido una jerarquización en torno a las corporalidades, excluyendo y desechando a aquellos cuerpos que no llegan a cumplir con las expectativas del modelo dominante.

La normalidad, en este sentido, tiene relación con un imaginario estético, funcional y político que ha sido construido desde las desde el poder económico, político y cultural (Uhart, 2004); estos parámetros de normalidad estarían asociados a la funcionalidad que los cuerpos pueden tener para el sistema, y no solamente desde la perspectiva de la productividad económica, sino también, desde una perspectiva del discurso racial, étnico, moral y cultural (Blas Lahittle y Fitte, 2007).

Pero, ¿cómo se ha logrado normalizar un tipo de cuerpo? y, sobre todo, ¿cómo se ha logrado colocar en el imaginario colectivo una idea homogeneizada de estética? Pues, a través de las narraciones que se han elaborado y transmitido históricamente; narraciones que, como sostienen López Hermida e Ibieta Illanes (2013), son formas de comunicación que convirtiendo a la persona emisora en un creador de un mensaje, que, al ser recibido por la o el usuario, moviliza, seduce, identifica.

Sin embargo, los relatos no son inocentes, sino que obedecen a intereses de clase, a una visión determinada de la sociedad, por lo que, las narraciones se convierten en dispositivos de ideologización. En la era de la información, son los medios de comunicación, a través de la publicidad, los que se encargan de transmitir el relato de los cuerpos y su estética normalizada. Es en la publicidad en donde se acentúan las características de la femineidad y la masculinidad y, además, de estilos de vida que se reflejan en un cuerpo-discursivo que tiene que ser vigilado constantemente.

Esto produce la imposición de una narrativa única sobre la corporalidad, que, a partir de las imágenes transmitidas por la publicidad, crea una clasificación de cuerpos masculinos y femeninos, que están jerarquizados por su apego a los parámetros del cuerpo modelo (Uhart, 2004). López y Gauli Pérez (2000), plantean que, por ejemplo, los regímenes políticos tienden a caracterizar los cuerpos de los 
buenos ciudadanos, así como también a caracterizar los cuerpos de los enemigos del Estado (anormales, enfermos psiquiátricos, terroristas, extranjeros), teniendo como objetivo el trazo de pautas de comportamiento modelo, es decir, una pretensión de homogeneización y desindividualización.

Dentro de estos esfuerzos por normalizar los cuerpos, el aporte de la narrativa médica ha sido fundamental a través del discurso médico que consolida una visión de un cuerpo normalizado y estéticamente aceptable (Foucault, 2006), provocando que la mayoría de los cuerpos enfermos, o considerados anormales, sean excluidos, o incluso invisibilizados. De esta manera, la medicina se convierte en una justificación para la publicidad de cuerpos normalizados bajo la estética de las clases dominantes.

La publicidad, que transmite y naturaliza una imagen homogénea de cuerpo, ha incluido en sus discursos las narrativas que relacionan a los cuerpos bellos con los cuerpos sanos, llevándonos a pensar que los cuerpos enfermos, necesariamente, no son estéticos, creando una narrativa de la enfermedad y la anormalidad. Según León (2012), la enfermedad supone un exilio, pues las presiones sobre el cuerpo son tales, que los individuos con cuerpos medicados, prefieren la soledad y el encierro a la constante exclusión.

Las narrativas que colocan al cuerpo saludable y pulcro como la única forma de corporalidad viable, no solamente se alimentan del enfoque médico, sino también de las categorías de etnia y clase social, pues más allá de la salud, lo que prima en las narrativas que la publicidad construye a partir de la categoría de salud, es un modelo de cuerpo único, étnicamente homogéneo, y funcionalmente apropiado para determinadas actividades relacionadas con la productividad y la división social del trabajo. Lo que las narraciones publicitarias, basadas en el discurso médico de lo sano y lo enfermo han colocado en el imaginario social, tiene que ver con colocar al cuerpo enfermo en la categoría de cuerpo en desuso, de cuerpo anormal, de cuerpo disfuncional (Blas Lahittle y Fitte, 2007).
Lo que este artículo pretende es reflexionar, a través de la etnografía y la fotografía, sobre la posibilidad de romper con la estética normalizadora del cuerpo sano, y dialogar con estéticas disidentes que entienden al cuerpo como un espacio de lucha y resignificación de la enfermedad. Además, se busca reflexionar sobre los intentos de construir una narrativa del cuerpo enfermo desde un enfoque de inclusión, utilizando la fotografía como herramienta de visibilización.

\subsection{Estado del arte}

Los trabajos que se vienen realizando, en el siglo XXI, sobre las nuevas narrativas de la salud son variados, enfocándose algunos en la necesidad de un nuevo relato entre pacientes y médicos, utilizando la compasión como eje central del discurso médico; $\mathrm{y}$, otros trabajos, que abordan la reflexión sobre la narración construida a través de la publicidad, de una estética normalizadora para el consumo médico.

Entre las investigaciones que tienen como eje central a la construcción de estereotipos, se encuentra el realizado por María López y Juan Gauli Pérez (2000), en el que analizan de qué manera se construyen los imaginarios sobre los cuerpos en la sociedad del siglo XXI, cuáles son los estereotipos que guían la construcción constante de los cuerpos masculinos y femeninos, y de qué forma los medios de comunicación limitan o contribuyen a estos procesos.

Héctor Blass Lahitte y Ana Leticia Fitte (2007), realizan un trabajo antropológico sobre género y enfermedad, en la que plantean que el cuerpo femenino ha sido tratado de acuerdo a una necesidad estética que tiene que cumplir con normas y características que una sociedad machista ha implementado, buscando en los cuerpos diferentes las razones para justificar y legitimar una estructura de género patriarcal. Esta investigación tiene como objetivo analizar la forma en la que la menopausia es tratada en la publicidad del cuerpo femenino. 
Denise León (2012), realiza una investigación sobre los cuerpos heridos, en la que plantea que la mayoría de los cuerpos enfermos son excluidos de los espacios de socialización por no cumplir con los parámetros de normalidad. Sin embargo, este trabajo explora las posibilidades de la poesía como herramienta para resignificar a los cuerpos heridos, descubrir sus padecimientos, y volverlos el eje central del debate médico-cultural.

López-Hermida e Ibieta Illanes (2013), trabajan sobre las nuevas narrativas en comunicación de la salud, reflexionando sobre la forma en la que el ciudadano tiene que enfrentarse al cúmulo de información referente a su cuerpo, colocando a la publicidad como un agente provocador de riesgos, pues genera procesos de información perjudicial al momento de identificar y tratar enfermedades.

Martha Gaviria (2015), realiza un trabajo sobre la narrativa en salud, partiendo de los postulados de Paul Ricoeur, y proponiendo una metodología para el análisis de las narrativas de la salud, utilizando como técnica la entrevista conversacional, la autobiografía y la interpretación hermenéutica. El objetivo de esta investigación es mirar de qué manera los narradores aportan, desde su vida y experiencia, a la construcción de una narrativa vinculada a los fenómenos de la salud y la vida.

Presentamos los fundamentos gnoseológicos, ontológicos, epistemológicos y metodológicos de una aproximación de investigación narrativa de tipo autobiográfico regido sobre la fenomenología hermenéutica de Paul Ricoeur. Esta es una propuesta novedosa para el campo de la investigación narrativa en salud que se caracteriza por los siguientes rasgos distintivos: 1) la entrevista conversacional representa una ruta idónea para la construcción de relatos que permiten la preservación de la memora de la memoria; 2) la autobiografía es el resultado de la memoria singular de recuerdos, silencios y olvidos, entretejida con otras memorias de personas contemporáneas, predecesoras y sucesoras; 3) la interpretación, que se despliega en el marco hermenéutico que se efectúa sobre la comprensión de las acciones narradas.
Monserrat Pulido-Fuentes (2016), realiza una investigación sobre las narrativas presentes en los pacientes de la Amazonía ecuatoriana, analizando de qué manera entienden las comunidades Achuar el sistema médico occidental y sus prácticas sobre la salud. La importancia de este trabajo radica en la discusión sobre las tensiones entre las narrativas en torno a cómo se entiende la salud desde los diversos contextos, y cuál es la conexión con la intersubjetividad de sus habitantes.

Carlos Rojas Jiménez (2017), investiga la forma en la que las narraciones se convierten en una herramienta fundamental para comprender al paciente, para explorar la enfermedad en su contexto social, y colocar, por tanto, a la compasión como un valor esencial a la hora de buscar una relación empática entre paciente y médico.

Estas investigaciones sirven como punto de partida para nuestra investigación, pues permiten colocar al cuerpo como un sujeto de debate político e ideológico, y a la salud como un dispositivo ideológico dentro de la construcción de jerarquías corporales relacionadas con la clase y la etnia.

2.

\section{Metodología}

El trabajo de investigación tuvo un enfoque cualitativo, utilizando a la etnografía como encuadre metodológico, que en términos de Restrepo (2018), permite una descripción de variados aspectos de la vida social, así como interpretaciones que se alimentan de los contextos propios de la investigación. El objetivo de utilizar a la etnografía como metodología, tiene que ver con la pretensión de realizar una descripción densa sobre la relación entre estética normalizada y enfermedad crónica, así como una interpretación sobre el discurso estético que tiene las personas con enfermedades crónicas que fueron entrevistadas.

Este trabajo de investigación fue realizado en la ciudad de Cuenca, Ecuador, con una muestra de 15 personas que tienen enfermedades crónicas que son visibles en su cuerpo. El tratamiento de la información 
fue realizado a partir de la firma de consentimientos informados (tanto para la entrevista como para las fotografías y su uso para la investigación y publicación) que nos permitieron conversar con ellos y ellas, y, además, construir una narración corporal a través de la fotografía.

Entre las técnicas de investigación aplicadas están la entrevista a profundidad, los grupos focales, y la fotografía. En cuanto a las entrevistas a profundidad, se realizaron 15 entrevistas semi estructuradas que duraron entre una y dos horas cada una, permitieron un diálogo sobre tópicos generales de la enfermedad, y cuestiones específicas referentes a la estética del cuerpo. Sobre los gurpos focales se aplicaron 5, y estuvieron estructurados con diversos actores de esta investigación, cuyo eje central era la discusión en torno a la estética de sus cuerpos, el dolor físico y relación entre enfermedad y vida cotidiana. Los grupos focales fueron llevados a cabo con la intervención previa de los investigadores, quienes iban moderando la discusión a través de guion semi estructurado.

Finalmente, la fotografía como técnica de investigación etnográfica, ha sido tomada como técnica y como objeto de análisis, pues, la aplicación de la fotografía en este trabajo de investigación permitió el diálogo entre la y el investigadores y el o la interlocutora, a través de la fotografía se pretendió establecer un diálogo que no fue posible a través de las entrevistas, y que tiene que ver con la forma en la que los y las interlocutoras representan su enfermedad en su cuerpo, cómo muestran un discurso a través de lo que su cuerpo es para ellos y ellas, y de qué manera se puede construir una narrativa del cuerpo enfermo.

La elección de la metodología y las técnicas, está alineada con un enfoque hermenéutico, en el que se ubica al investigador en un espacio cercano al fenómeno social, pudiendo entender las narrativas desde el lugar en el que se dan los hechos (Pulido-Fuentes, 2016). El análisis de la intersubjetividad es importante para entender cuál es la percepción que los interlocutores tiene sobre sus cuerpos y la enfermedad, cuáles son los significados que la sociedad da a la forma en la que la persona entrevistada afronta la enfermedad, y cuáles son las posibles resignificaciones que se pueden realizar a partir de la construcción de una narrativa de la enfermedad.

\section{3.}

\section{Resultados}

Los resultados obtenidos, en cuanto a la construcción de narrativas contra-hegemónicas a través de la fotografía, tienen que ver con los métodos aplicados. Los resultados van a ser expuestos según tres momentos: dos momentos que fueron identificados en las entrevistas a profundidad y que tienen que ver con la forma en la que las personas participantes miraban su cuerpo al momento de enterarse del padecimiento de la enfermedad crónica, y el segundo tiene que ver con la forma en la que las personas participantes miran su cuerpo luego de un proceso de tratar y comprender su enfermedad. El tercer momento tiene que ver con la construcción de una narrativa del cuerpo enfermo a través del trabajo fotográfico.

\subsection{Primer momento. Descubrir la enfermedad}

\section{Entrevista a profundidad}

Identificación de la presencia de una estética normalizada en el imaginario colectivo: Las entrevistadas y los entrevistados identifican que, en el momento de enterarse de su padecimiento, uno de los cuestionamientos principales tenía que ver con la estética de su cuerpo. Todos y todas concuerdan en que les preocupaba la forma en la que podría "dañarse" su cuerpo, y las repercusiones que eso podría tener en su vida social.

La presencia de una estética normalizada en el imaginario colectivo se evidencia en las preocupaciones que las entrevistadas y los entrevistados tienen en torno a su cuerpo, pero en la relación con otras personas. Por ejemplo, las complicaciones que manifiestan tienen que ver con el trabajo, las aulas de clases, y la relación de pareja.

Esto evidencia que en las actividades cotidianas se está normalizado una imagen de un cuerpo sano y normal, un cuerpo que no tenga llagas, que no tenga manchas, un cuerpo que no sienta dolor, y que pueda cumplir las funciones dentro de la división social del trabajo. 


\section{Grupos focales}

Existe una relación entre la narrativa creada por la publicidad basada en el cuerpo saludable, y la normalización de una estética dominante que obedece a categorías de clase y etnia: Las entrevistadas y los entrevistados sostienen que enfrentarse a la enfermedad por primera vez, les hizo pensar que sus cuerpos ya no serían normales. El tratamiento, el dolor, las secuelas de la enfermedad en su piel les hizo pensar que su cuerpo sería excluido porque la sociedad no está acostumbrada a lo que no corresponde a un cuerpo estéticamente normalizado.

Comentan en la discusión de los grupos focales, que, al estar expuestos toda la vida a publicidad sobre el cuerpo, sobre el cuidado, sobre lo sano, interpretaron que un cuerpo es bello solamente cuando cumple con estas características. Además, sostienen que la publicidad muestra cuerpos que se convierten en los modelos a seguir, pero que es muy difícil llegar a cumplir con esos parámetros.

Esto refuerza la idea de que se ha normalizado un cuerpo estéticamente bello como sinónimo de salud, y que, además, la pulcritud de un cuerpo, en cuanto a la falta de llagas o manchas, también define la belleza.

Finalmente, la construcción de un cuerpo modelo está pensado desde parámetros de clase y etnia que obedecen a la estructura social blanco-mestiza imperante, y a una división social del trabajo propia de esta estructura.

\subsection{Segundo momento: Tratar y comprender la en- fermedad}

\section{Entrevista a profundidad}

Al momento de tratar de comprender la enfermedad se forman estéticas disidentes: en los relatos de las entrevistadas y los entrevistados existe un escenario común, la resignación como paso a la comprensión de la enfermedad. Al hablar de enfermedades crónicas, estamos hablando de enfermedades que no van a desaparecer, por tanto, el tratamiento es la única alternativa para llevar una vida más o menos normal, nos vuelven a recalcar los y las interlocutoras. El tra- tamiento de las enfermedades les permite mitigar el dolor, y esto provoca un proceso de resignación en torno a la enfermedad, resignación que luego viene acompañada de una comprensión de su situación, y la comprensión de lo que significa eso individualmente y socialmente.

Lo interesante de la comprensión de la enfermedad, es que se produce una ruptura con la narrativa del cuerpo estéticamente bello, y se comienza a construir una estética de la enfermedad, que tiene como centro la salud. Es decir, al hablar de salud no hablamos solamente del cuerpo sano como modelo estético, sino que abordamos a la salud como un proceso de interacción necesaria, en el que los cuerpos que sufren enfermedades necesitan de las relaciones sociales para combatir la enfermedad.

De esta manera, el proceso de comprensión de la enfermedad permite la creación de estéticas disidentes que no quieren acoplase al modelo normativo, y más bien, lo desafían.

\subsection{Tercer momento: Construcción de una narrativa del cuerpo enfermo a través del trabajo fotográfico.}

\section{Fotografía}

Construcción de una narrativa del cuerpo enfermo a través del trabajo fotográfico: la aplicación de la fotografía en este trabajo de investigación tenía como objetivo la construcción de una narrativa alternativa a la planteada por la publicidad basada en la estética del cuerpo sano. 


\section{Fotografía 1.}

Participante 1

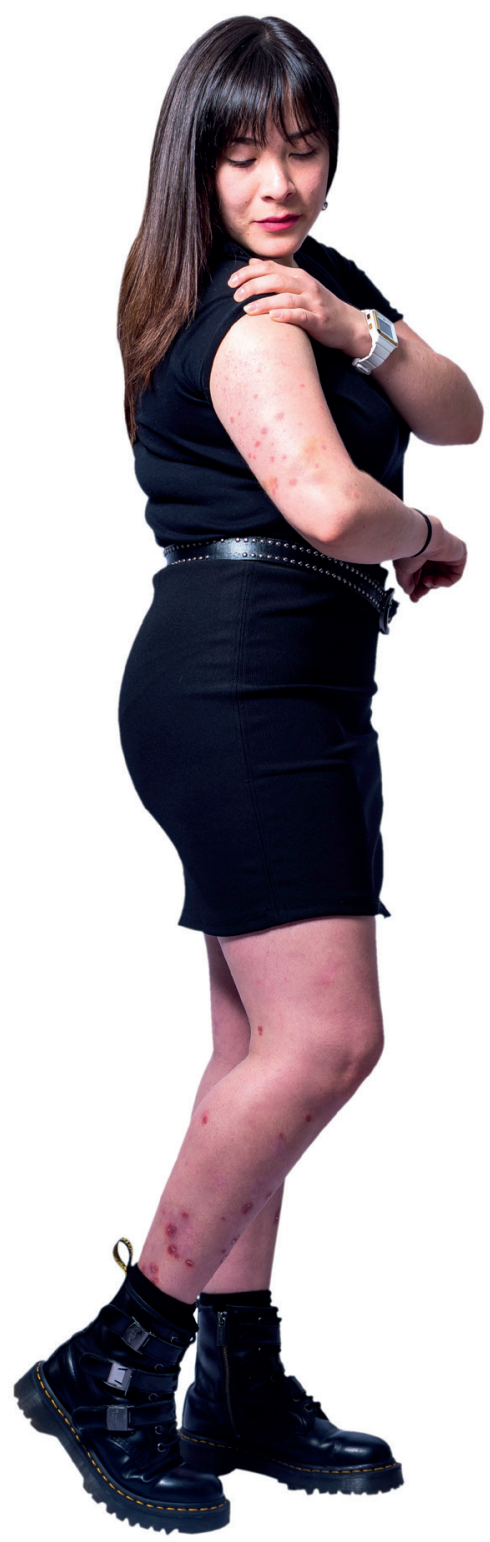

Fuente: Neira Ruiz, S. (2020). [Participante 1 de la investigación: psoriasis].

La secuencia fotográfica muestra cuerpos bellos.

\section{Fotografía 2.}

Participante 2

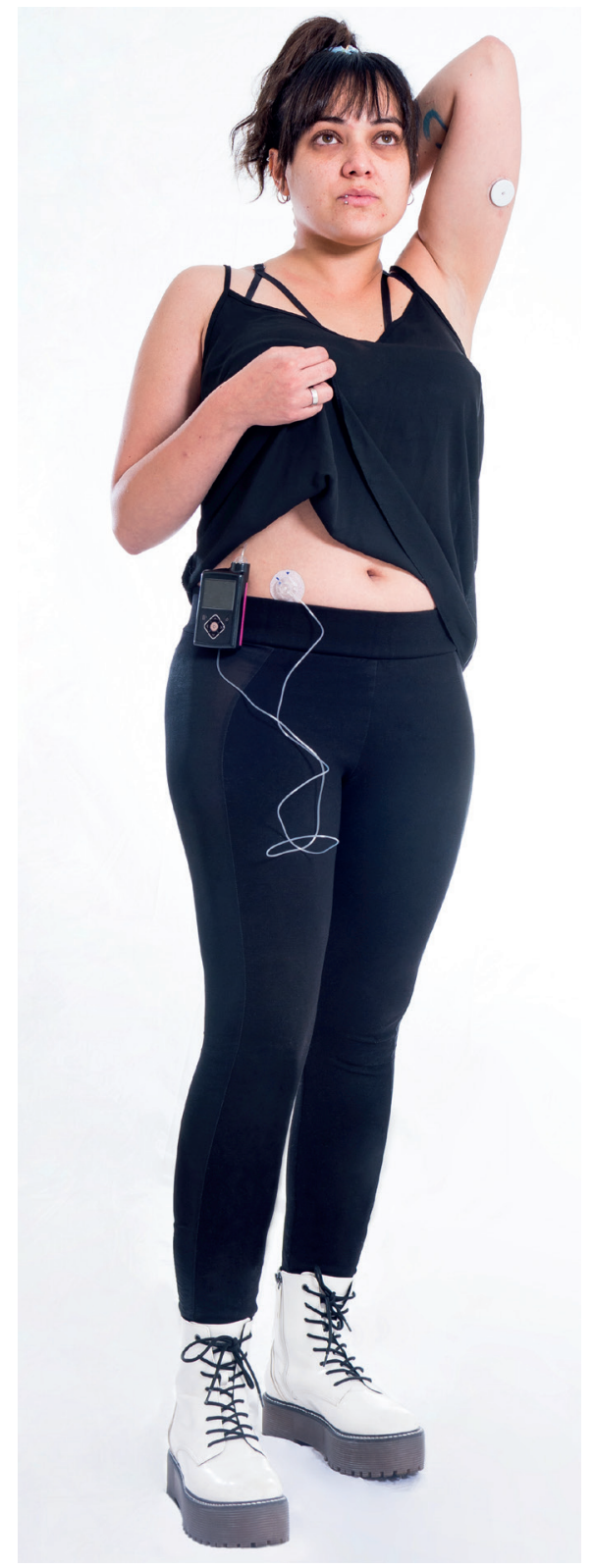

De Neira Ruiz, S. (2020). [Participante 2 de la investigación: diabetes].

Las fotografías rompen con el esquema de estética normalizada, y construyen una narrativa de la estética del cuerpo enfermo a través de la fotografía. 


\section{Fotografía 3.}

Participante 3

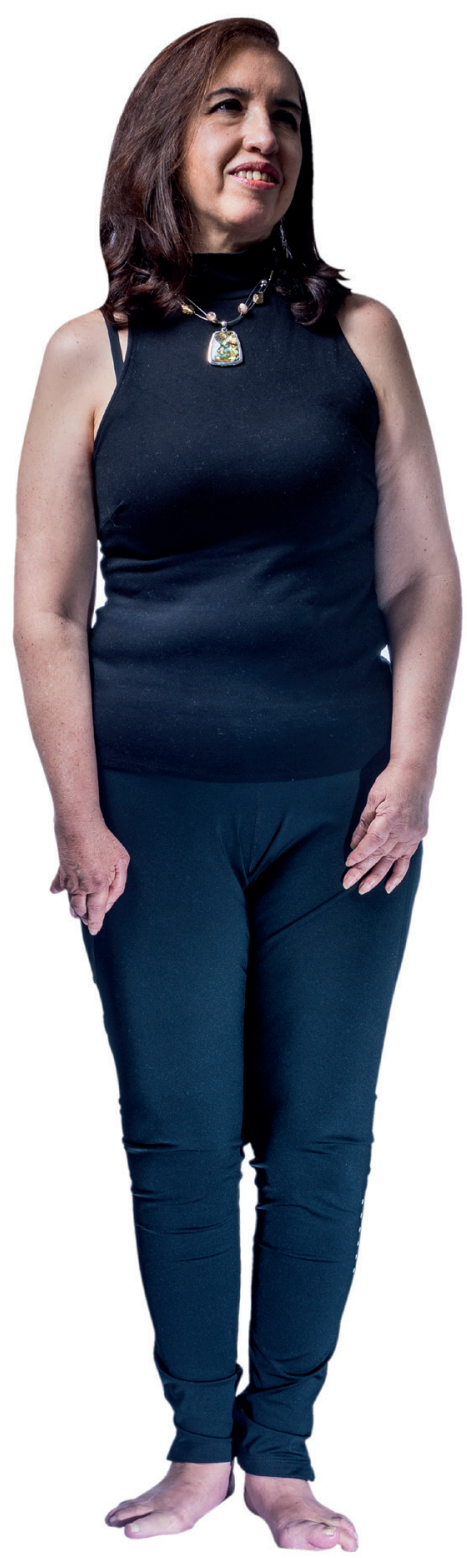

De Neira Ruiz, S. (2020). [Participante 3 de la investigación: artritis juvenil].

Las poses por las que optan los participantes, son un juego de contra-poder a la estética normalizada y vendida por la publicidad.

\section{Fotografía 4 .}

Participante 4

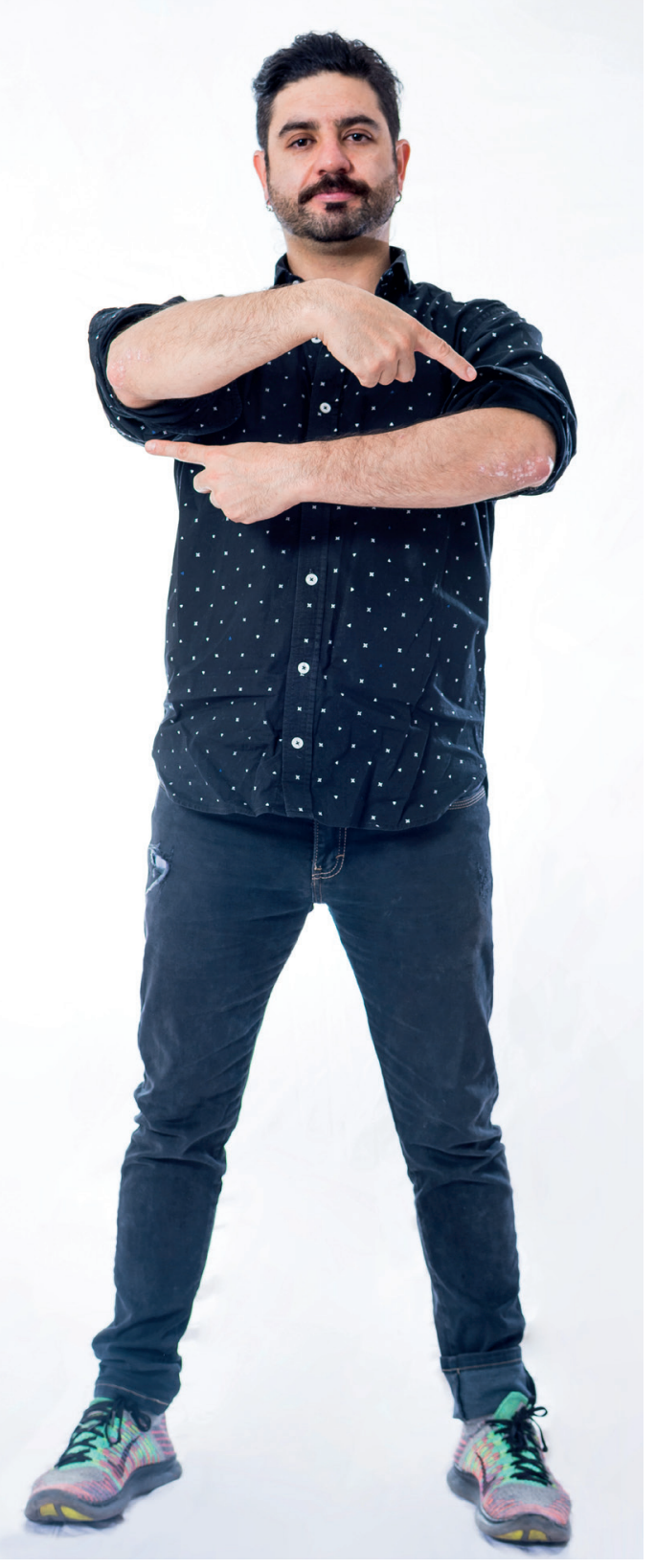

De Neira Ruiz, S. (2020). [Participante 4 de la investigación: psoriasis]. 


\section{4.}

\section{Discusión}

La construcción de nuevas narrativas corporales que vayan más allá del discurso de las narrativas normalizadas del cuerpo estéticamente sano, nos da una nueva posibilidad de conocimiento sobre los sentires de las personas que padecen enfermedades crónicas, nos permite conocer nuevos relatos sobre estéticas excluidas históricamente, aporta nuevas perspectivas a la discusión sobre el cuerpo, que se pierden al momento de relacionar a la salud con la estética homogeneizadora. Este resultado de la investigación está en consonancia con lo que Jiménez (2017) plantea sobre la posibilidad de conocer al otro a través de la narración y sobre la necesidad de explorar los relatos que nos hacen más empáticos con las personas que han sido invisibilizadas en el relato oficial.

Entender que los cuerpos enfermos y heridos pueden generar narrativas alternativas nos lleva a plantear la necesidad de diálogo entre los diversos tipos de corporalidad, y permite mitigar el silencio al que han sido sometidas las personas que sufren enfermedades. Visibilizar los diversos relatos de contextos diferentes nos lleva a la comprensión de que los cuerpos enfermos también son parte de la comunidad. Esta construcción de narrativas alternativas genera lo que Léon (2012) llama "ruptura del mito de la corporalidad única", mostrando una gama diversa de comportamientos y discursos que pueden ser parte de la estética de los cuerpos. A través de la fotografía, como técnica de construcción de un relato alternativo, se trata de aportar en la visibilización de cuerpos que han sido históricamente excluidos, como es el caso, de los cuerpos que sufren enfermedades crónicas visibles.

Pulido-Fuentes (2016) plantea que existen sensaciones en las personas enfermas que son socialmente construidos, y que obedecen a la forma como la sociedad mira el cuerpo enfermo. Los relatos de los y las participantes de esta investigación ejemplifican lo que Pulido-Fuentes plantea, pues las miradas que la sociedad tiene sobre los cuerpos enfermos condiciona a la mirada que las personas que padecen enfermedades crónicas tienen sobre sus propios cuerpos. Por ejemplo, Susana, en una de las entrevistas, nos comentaba que, "existe una incomprensión sobre la enfermedad, que socialmente, nos vemos obligadas a esconder nuestros cuerpos" (Entrevista a Susana, 2019). Pensar que la sociedad acepta solamente un tipo de cuerpo profundiza el silencio y la auto-exclusión.

He aquí la necesidad de construir una narrativa del cuerpo enfermo que pueda hacer frente a la idea de la existencia de un solo tipo de estética corporal asociada a la idea publicitada sobre el cuerpo sano que, además, está cargada de componentes clasistas y étnicos. Presentar, visualmente, a través de la fotografía, una secuencia de imágenes que muestren la posibilidad de ruptura con el paradigma corporal normalizado abre camino para reflexionar y pensar en la construcción del lenguaje publicitario en el que se muestra al cuerpo sano como el único camino posible de la construcción de una estética aceptable.

La inexistencia de otros cuerpos en la narrativa de la salud imposibilita la comprensión de las enfermedades como un proceso que puede ser tratado y vivido desde el auto-cuidado y el auto-conocimiento. López y Gauli Pérez (2000) plantean que la publicidad cada vez intensifica más el discurso hegemónico sobre qué tipos de cuerpos son aceptados y qué cuerpos son invisibilizados, lo que se ha podido comprobar a través de los relatos de las personas que participan en esta investigación. Los temores que están ligados a las enfermedades crónicas, tienen que ver con la forma en la que van a ser aceptados esos cuerpos marcados por la forma en la que pueden ser excluidos de las dinámicas sociales.

La posibilidad de mostrar a los cuerpos que padecen enfermedades crónicas a través de la fotografía ofrece una ruptura con el modelo corporal dominante que se ha impuesto. López y Gauli Pérez (2000) plantean que la belleza normalizada de los cuerpos considerados sanos, es solamente una interpretación, cuestión que se encuentra presente en los relatos de las entrevistadas y los entrevistados, cuando plantean que, al vivir un proceso de resignación de la enfermedad, y, por lo tanto, de comprensión de su cuerpo, comienzan a interpretar que cabe 
la posibilidad de belleza en los cuerpos que padecen enfermedades crónicas. Juana, al conversar con nosotros sobre la forma en la que vive su enfermedad, nos comentaba que, "al inicio era difícil, porque tenía la sensación de que no era bella, de que mi cuerpo con llagas era feo, pero luego me di cuenta que no es así, que puede ser hermosa de esta forma, y ahora me siento bien con mi cuerpo" (Entrevista a Juana, 2019).

La construcción de una narrativa disidente y alternativa de los cuerpos que padecen enfermedades crónicas nos enfrenta a la posibilidad de entender el cuerpo y su estética desde otros enfoques, nos permite entender la multiplicidad de discursos que se expresan a través del cuerpo y nos entrega un espacio de reflexión en torno a los procesos de publicidad de los cuerpos sanos y normalizados. Una narrativa del cuerpo enfermo, que no solo se presenta de manera teórica, sino que también aporta desde un enfoque visual y artístico, puede aportar al debate de la de construcción de modelos de corporalidad homogeneizados por los grupos que ejercen el poder político, económico, religioso y cultural.

\section{5.}

\section{Conclusiones}

El análisis realizado en este artículo permite seguir reflexionando y discutiendo en torno al cuerpo, la estética y la enfermedad. La metodología aplicada permitió generar algunas reflexiones sobre la normalización de un modelo de cuerpo único y su relación en una dicotomía de salud enfermedad. Permitió discutir de qué forma la normalización de estéticas corporales podrían estar relacionadas con narrativas en salud que han provocado la homogeneización de una visión determinada sobre los cuerpos, y, por lo tanto, la exclusión de todas aquellas corporalidades que no se adapten a estos parámetros.

Se ha contribuido, a través del relato fotográfico y la reflexión teórica, al debate sobre la posibilidad de construir estéticas alternativas a partir de la disidencia de los cuerpos que padecen enfermedades crónicas. Las técnicas aplicadas en esta investigación permitieron llegar a resultados que son un punto de partida para continuar el trabajo de investigación sobre la construcción de las corporalidades dominantes y las corporalidades excluidas.

Sin embargo, la localización de esta investigación está centrada en la ciudad de Cuenca-Ecuador, lo que se convierte en un limitante al momento de generalizar los resultados, pero, al mismo tiempo, se convierte en una oportunidad para ser un punto de partida de futuras investigaciones en el tema, que puedan aplicar esta investigación en otros lugares, con contexto culturales e históricos distintos.

El análisis de las categorías de clase y etnia han sido abordadas de una manera muy breve, lo que nos coloca frente a la posibilidad de profundizar estas relaciones en trabajos posteriores, pues la construcción de narrativas corporales homogeneizadoras necesariamente está pensada desde constructos ideológicos que miran a los cuerpos como dispositivos para perpetuar un orden social establecido.

De esta manera, este artículo ofrece un acercamiento a la reflexión teórica y metodológica sobre la posibilidad de crear narrativas que se opongan a los modelos normativos y normalizados, y que ofrezcan la necesidad de entender al otro marginado y excluido, no desde la superioridad, sino desde la comprensión de que mi cuerpo discursivo es solamente una forma de construir una corporalidad estética.

Agradecimiento: A Santiago Neira Ruiz por realizar todas las fotografías que han acompañado esta investigación.

6.

\section{Referencias}

Blas Lahittle, H.; Fitte, A. (2007). Antropología y construcciones de género: publicidades gráficas y menopausia. Revista de Ciencias Sociales (CI), 19, segundo semestre, pp. 41-57. https://www.redalyc.org/pdf/708/70801903.pdf 
Butler, J. (2012). Cuerpos que importan-sobre los limites materiales y discursivos del "sexo". Paidos.

Deleuze, G. (1989): ¿Qué es un dispositivo?, en: Michel Foucault, filósofo, Gedisa, pp. 155-163.

Foucault, M. (2006). El nacimiento de la clínica. Siglo XXI.

Jiménez Guzmán, C. (2018). Sujeto disciplinado, sujeto normalizado, sujeto subjetivado. https://repository.upb.edu.co/ handle/20.500.11912/4888

Kottow, A. (2016). Patologías Deconstructivas: cuerpos enfermos y razón moderna en la literatura chilena del siglo XIX. República de la Salud. Fundación y ruinas de un país sanitario. Chile, siglos XIX y XX. En: Araya, Claudia, pp. 17-31.

Ledón Llanes, L. (2011). Enfermedades crónicas y vida cotidiana. Revista cubana de salud pública, 37(4), pp. 488-499.

https://doi.org/10.1590/

$\underline{\text { S0864-34662011000400013 }}$

León, D. (2012). El cuerpo herido. Algunas notas sobre poesía y enfermedad. Telar. 10. pp. 53-74. https://ri.conicet.gov.ar/handle/11336/39883

López, M.; Gauli Pérez, J. (2000). El Cuerpo imaginado. Revista Complutense de Educación, vol.11, núm. 2, pp. 43-57.

López-Hermida, A. y Ibieta Illanes, N. (2013). Nuevas narrativas en Comunicación de Salud: El storytelling y la conquista emocional del paciente. Anuario Electrónico de Estudios en Comunicación Social "Disertaciones", 6 (2), Artículo 3. https://ng.cl/gndtj
Melguizo, R. C. (2014). Evolución del concepto de discapacidad en la sociedad contemporánea: de cuerpos enfermos a sociedades excluyentes. Praxis sociológica, (18), pp. 155-175. https://n9.cl/a5qp2

Orobitg, G. (2014). La Fotografía en el Trabajo de Campo: palabra e imagen en la investigación etnográfica. Quaderns-e de l'Institut Català d'Antropologia, (19 (1)), pp. 3-20. https://n9.cl/z2wk

Pedraza, Z. (2009). Derivas estéticas del cuerpo. Desacatos. 30, pp. 75-88. https://ng.cl/v9wd

Pulido-Fuentes, M. (2016). Salud y Enfermedad desde la narrativa de los pacientes en la Amazonía Ecuatoriana. Revista San Gregorio, No.13, Edición Especial Congreso CEISAL pp. 6-19. https://doi.org/10.36097/rsan.voi13.307

Rangel Flores, Y. (2014). El Modelo Histórico-narrativo, una propuesta para estudiar el riesgo en salud. Index de Enfermería, 23(1-2), pp. 7074 .

https://doi.org/10.4321/

$\underline{\mathrm{S} 1132-12962014000100015}$

Restrepo, E. (2018). Etnografía: alcances, técnicas y éticas. Universidad Nacional Mayor de San Marcos.

Rosas Jiménez, C. (2017). Medicina Narrativa: El Paciente como "texto", objeto y sujeto de la compasión. Acta Bioethica, 23 (2), pp. 351-359.

http://dx.doi.org/10.4067/

S1726-569X2017000200351

Sánchez, G., Laza, C., Estupiñán, C., \& Estupiñán, L. (2014). Barreras de acceso a los servicios de salud: narrativas de mujeres con cáncer de mama en Colombia. Revista Facultad Nacional de Salud Pública, 32(3), pp. 305-313.

https://ng.cl/2vr2 